\title{
CHAMP gravity field recovery using the energy balance approach
}

\author{
Ch. Gerlach ${ }^{1}$, N. Sneeuw ${ }^{2}$, P. Visser ${ }^{3}$, and D. Švehla ${ }^{1}$ \\ ${ }^{1}$ Institut für Astronomische und Physikalische Geodäsie, Technische Universität München, Germany \\ ${ }^{2}$ Department of Geomatics Engineering, University of Calgary, Canada \\ ${ }^{3}$ Delft Institute for Earth Oriented Space Research, Delft University of Technology, The Netherlands
}

\begin{abstract}
Since the early days of satellite geodesy energy balance based methods for gravity field determination have been considered. If non-conservative forces are known the Hamiltonian along the orbit is a constant of the motion. Thus the gravity field can be determined if position and velocity of the satellite are known and accelerometer measurements are available to model the non-conservative part. CHAMP is the first satellite that provides the user with those three kinds of data nearly continuously. Numerical investigations using real CHAMP data are presented to show the feasibility of the method. Using a semi-analytical approach the gravity field can be determined efficiently by a 2D-Fourier method. Those fast computations also give way to application of the method not only to a full gravity field recovery but also, e.g. for quick-look and validation of SST observations for satellite missions like CHAMP, GRACE or GOCE. The method can also be used for estimation of accelerometer calibration parameters.
\end{abstract}

Key words. gravity field, energy balance, Jacobi-integral, non-conservative forces, accelerometer calibration, CHAMP

\section{Introduction}

In 1836 the mathematician C. G. J. Jacobi presented an integral of motion for the restricted three-body problem (see Jacobi, 1836). There a body of negligible mass (like a comet or a satellite) moves within the gravity field of a pair of point masses, which are rotating on circular orbits around their common center of mass (like the sun and a planet). This so called Jacobi integral was widely used in astronomy. One of the application was, e.g. to identify comets independent of their orbital elements, which can be strongly affected by planetary disturbances - an idea proposed by Tisserand (see e.g. Guthmann, 2000).

As stated by Hotine and Morrison (1969), a pair of rotating point masses is a special case of a rotating rigid body.

Correspondence to: Ch. Gerlach (gerlach@bv.tum.de)
Therefore Jacobi's integral can be generalized to the case of an earth orbiting satellite. Then it reads

$W(\boldsymbol{x})-\frac{1}{2} \dot{\boldsymbol{x}}^{2}=C$,

where $W$ is the gravity potential of the earth (sum of gravitational and centrifugal potential), $\boldsymbol{x}$ and $\dot{\boldsymbol{x}}$ are position and velocity of the satellite and $C$ is the so called Jacobi-constant. The second term on the left hand side is equal to the satellite's kinetic energy. Equation (1) holds in an earth fixed reference frame (CTS, conventional terrestrial system). It can also be derived from the satellite's equations of motion, as shown e.g. by Schneider (1967).

The geodetic application of Jacobi's integral was - as far as we know - for the first time proposed by O'Keefe (1957). He suggested to use the balance between gravity potential and kinetic energy to determine the gravitational potential of the earth by measuring the velocity of a satellite. More precisely speaking, potential differences can be derived because in Eq. (1) $C$ is unknown. But as only the zero-degree coefficient of a spherical harmonic expansion of the gravity field is affected, this shall be of no relevance for our further discussion. However for geodetic application Jacobi's integral, as given in Eq. (1), suffers from disregarding perturbations like tides or air resistance.

Jacobi's integral is formally equivalent to the law of energy conservation. Potential and kinetic energy of a satellite are equal to the first and second term on the left side of Eq. (1) and the constant $C$ corresponds to the Hamiltonian (total energy) of the mechanical system. In the late sixties Bjerhammar (1967) proposed - without derivation - such a formulation, based on energy considerations, to determine earth's gravitation. There he already included effects of sun and moon as well as corrections for air drag and solar radiation pressure. The latter are non-conservative forces and must be included in order to compensate for the loss of energy due to friction.

As theoretical basis to Bjerhammar's idea Hotine and Morrison (1969) investigated the integrals of motion of a satellite 
and generalized the formulas for the case of time dependent potential fields and non-conservative forces. They derived the energy integral both in an earth fixed as well as in a space fixed frame (CIS, conventional inertial system). The former is equal to Eq. (1), while the latter reads

$\frac{1}{2} \dot{\boldsymbol{x}}^{2}+W-\boldsymbol{\Omega} \boldsymbol{L}=C$.

There $\boldsymbol{\Omega}$ is the vector of earth's rotation and $\boldsymbol{L}$ is the satellite's angular momentum $\boldsymbol{L}=\boldsymbol{x} \times \dot{\boldsymbol{x}}$.

Equations (1) and (2) both imply that position and velocity of the satellite are measured continuously. Today this can be done, e.g. by high-low tracking from GPS satellites. Wolff (1969) proposed to apply the energy considerations to a pair of low orbiting satellites, making use of low-low satellite-tosatellite tracking (SST). The potential differences between the locations of the two satellites can be determined from the measured range rate between them.

Detailed numerical investigations on the feasibility of gravity field determination based on Jacobi's integral were performed by Reigber (1969), using simulated data. Due to the technique and quality of orbit determination at that time, the results were pessimistic and the method has not been used operationally until today. Still there have been theoretical and numerical simulation studies, e.g. by Ilk (1983), Jekeli (1999), Ilk (2000), Gerlach and Sneeuw (2000) or Visser et al. (2003).

Recently the feasibility of the method was tested using real data by Gerlach et al. (2003) and Howe and Tscherning (2003). Both groups made use of the rapid science orbits (RSO), provided by GeoForschungsZentrum (GFZ), Potsdam, for the CHAMP-satellite. The RSO is produced mainly for use in GPS radio occultation measurements, where the accuracy requirements are not that demanding. In contrast, as shown by Visser et al. (2003), the energy balance approach is highly sensitive to velocity errors. It is the aim of this paper, to increase the accuracy of the solution shown in Gerlach et al. (2003) by using a precisely determined orbit, which is more accurate than the RSO.

\section{Energy balance approach}

Jacobi's integral, as given in Eq. (1), is only valid in the absence of non-conservative forces and for an earth with constant gravity field (no temporal changes in gravitation and earth rotation). In reality additional terms must be taken into account to correct for solar radiation pressure, air drag, tides, etc. The applied corrections are based on energy considerations rather than on a rigorous derivation of a generalized Jacobi integral. That's why we call the method energy balance approach instead of using the term Jacobi integral.

The loss of energy $\Delta C$ due to non-conservative forces, like air drag or solar radiation pressure, is equal to the work done by this forces along the satellite's trajectory and can be com- puted by integrating the corresponding accelerations $\boldsymbol{a}$ along the orbit according to

$\Delta C=\int_{\boldsymbol{x}} \boldsymbol{a} \mathrm{d} \boldsymbol{x}$

In practice the gravity potential $W$ in Eq. (1) is not only induced by the earth. It also contains contributions of all third bodies like sun, moon or planets (direct tidal potential) as well as indirect tidal effects (solid earth tides, ocean tides, etc.) and further temporal variations of the gravitational field of the earth. Also the centrifugal potential is not constant due to changes in earth rotation. For short observation periods (like one or two weeks) we assume, that there are no temporal variations in the Earth's gravity field, so we can neglect those effects and only include tides. The direct tidal potential induced by the body $i$ is given by (see e.g. Torge, 1980)

$V_{i}=G M_{i}\left(\frac{1}{l}-\frac{1}{r_{i}}-\frac{r_{s} \cos \psi}{r_{i}^{2}}\right)$,

where $r_{s}$ and $r_{i}$ are the geocentric radii of satellite and body $i$, respectively, $l$ and $\psi$ are distance and geocentric angle between the two and $M_{i}$ is the mass of $i$. For the time span used in this paper (see Sect. 3) the direct luni-solar tides contribute to the potential at CHAMP altitude with up to around $6 \mathrm{~m}^{2} \mathrm{~s}^{-2}$. The indirect effects of solid earth and ocean tides are modelled according to the IERS conventions (McCarthy, 1996). They amount to up to around $1 \mathrm{~m}^{2} \mathrm{~s}^{-2}$ for the solid earth tides and around $0.3 \mathrm{~m}^{2} \mathrm{~s}^{-2}$ for the ocean tides. All other effects (pole tides, direct tidal potential of planets, etc.) are at least one order of magnitude smaller and are therefore neglected in the sequel.

Adding the loss of energy $\Delta C$ to the left hand side of Eq. (1) and splitting the potential $W$ into tidal potential $V_{t}$ (sum of direct and indirect tidal effects), centrifugal and gravitational potential of the earth we get for the disturbing potential $T$ of the earth

$T+C=\frac{1}{2} \dot{\boldsymbol{x}}^{2}-\frac{1}{2}(\boldsymbol{\Omega} \times \boldsymbol{x})^{2}-\Delta C-V_{t}-U$,

where the second term on the right hand side is the centrifugal potential and $U$ is the normal potential. This is the basic equation we use for determination of the earth's gravitational field. It allows to derive point values of disturbing potential along the orbit. In the following analysis these potential values are used as pseudo-observations based on the measurements $\boldsymbol{x}, \dot{\boldsymbol{x}}$ and $\boldsymbol{a}$. As already mentioned the energy constant $C$ is unknown. But because the disturbing potential fluctuates around zero we have simply introduced as approximate value for $C$ the mean of the time series on the right hand side of Eq. 5.

\section{Input data}

To derive the disturbing potential according to Eq. (5) orbit and accelerometer (ACC) data of GFZ's CHAMP satellite 
are used. CHAMP, launched in July 2000, is the first satellite mission that allows to apply the energy balance approach, because there is continuous high quality tracking of the satellite with GPS and the non-conservative forces are measured by accelerometry onboard CHAMP. Furthermore, using a reduced dynamic technique for orbit determination, the outcome of the computations are not only positions but also velocities. In fact these velocities give rise to further concern because they are highly correlated to the a priori gravity field, used in the orbit determination. This will be discussed later.

\subsection{Orbit data}

In this paper we have used an 11-days (20 to 30 May 2001) reduced-dynamic orbit (denoted TUM), determined by Švehla and Rothacher (2003) in the framework of the CHAMP Orbit Comparison Campaign. Within this 11 days CHAMP covers the earth quite homogenously, leading to a good sampling of the gravity field. Orbit data are given in CTS and provided in $30 \mathrm{~s}$ sampling over arcs of $24 \mathrm{~h}$. As a priori model for orbit computation the EIGEN-1S gravity field model (Reigber, 2001a) was used.

\subsubsection{Precise orbit determination}

The dynamic orbit was computed in a double-difference approach using carrier-phase GPS measurements only. One of the advantages of using double-differences is, that we get rid of all epoch-wise GPS and LEO clock parameters. The drawback is, that measurements from the IGS ground network have to be included. The main advantage of the doubledifference approach is, however, that ambiguity resolution can be done.

As mathematical model for dynamic precise orbit determination (POD) a normal least-squares adjustment was used with variational equations without making use of any filtering. The basic idea of this dynamic POD can be summarized in the following two-step procedure:

1. using a first set of kinematic positions (e.g. from code observations) as pseudo-observations all dynamic parameters are solved for and an a priori dynamic orbit is computed. The partial derivatives of the satellite position with respect to all orbit parameters are computed in this step by a simultaneous integration of the equation of motion and the variational equations;

2. using the carrier-phase measurements the a priori orbit is improved by estimating all orbital parameters and pseudo-stochastic pulses. More frequent setting up of stochastic pulses may be considered equivalent to modelling the air-drag with more parameters. That's why this approach is called reduced-dynamic POD. Air-drag densities are modelled using MSISE-90 (Hedin, 1991).

The accuracy of the dynamic positions, as compared to satellite laser ranging (SLR), is at the level of around $4 \mathrm{~cm}$ (RMS). The SLR residuals for the TUM-orbit are shown in Fig. 1. They were computed as the difference between

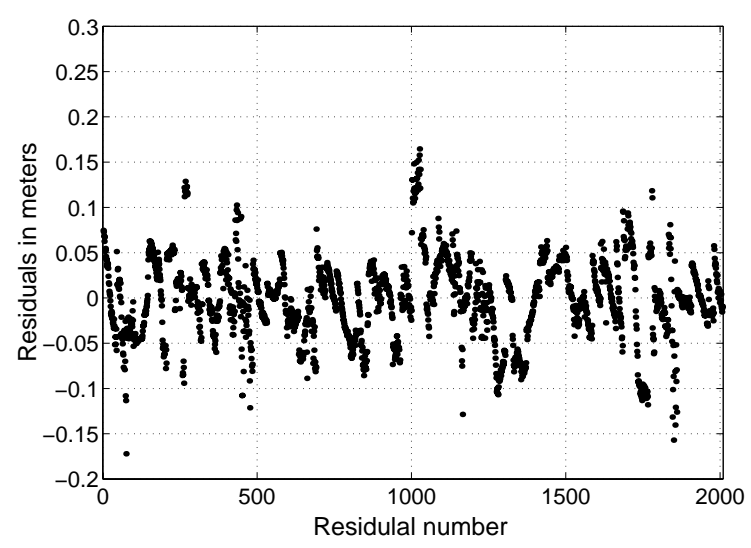

Fig. 1. SLR residuals for double-difference dynamic orbit over 11 days $(\mathrm{RMS}=44.3 \mathrm{~mm})$.
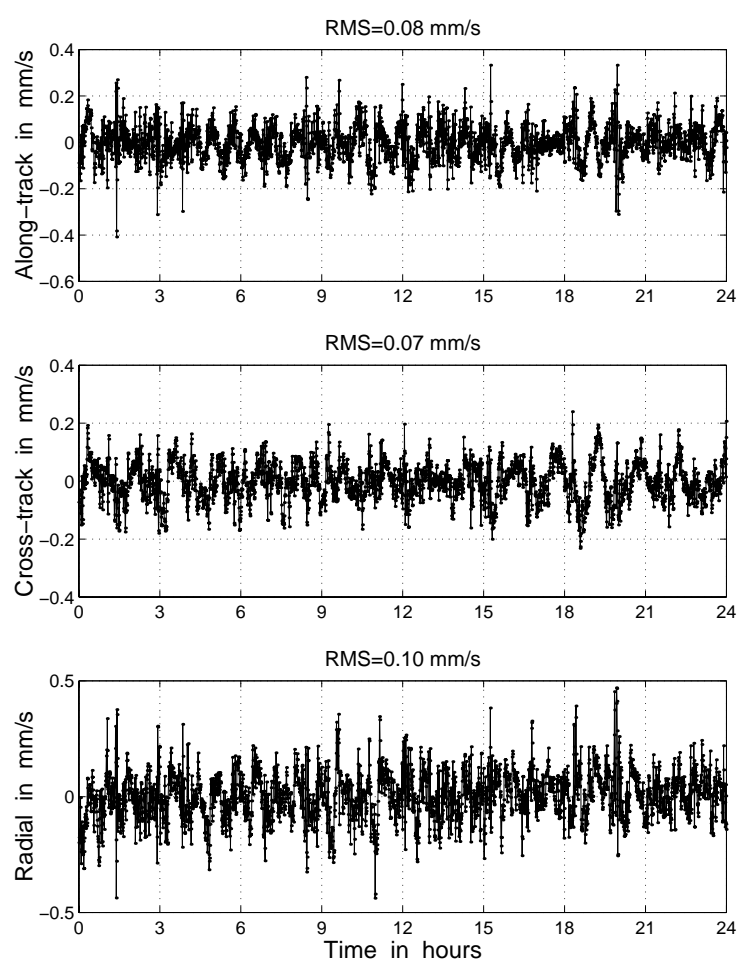

Fig. 2. Comparison of CHAMP dynamic velocities from CSR and TUM, day 148/2001.

the SLR measurements (corrected for the tropospheric delay with the Marini-Murray model) minus the distance between the SLR station and the GPS-derived orbit position. Altogether 2007 SLR residuals were obtained in this way using 69 daily station files from 17 SLR stations over 11 days.

In order to validate the velocities of the TUM-orbit, comparison between several POD centers was done, namely the Center for Space Research (CSR), Austin Texas and GFZ. Figure 2 shows the comparison with CSR dynamic velocities for May 28th, 2001 (day 148 of the year). It is worthwhile 


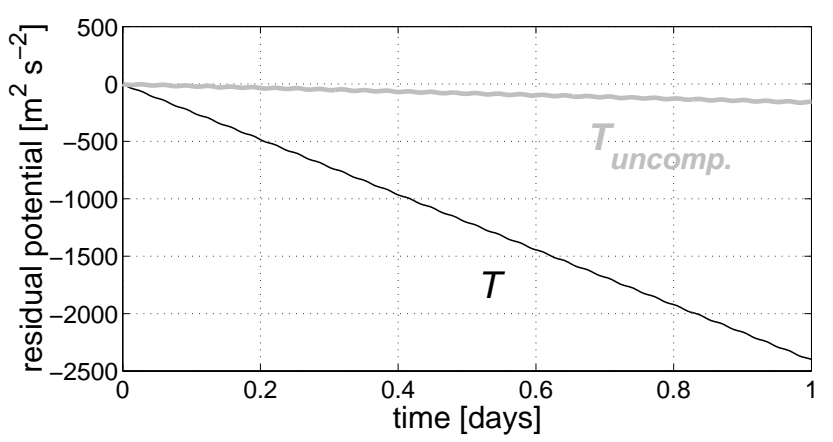

Fig. 3. Residual disturbing potential derived from energy integral with respect to geopotential model EIGEN-1S. $T$ is compensated for $\Delta C$, while $T_{\text {uncomp. }}$ is not.

noticing that CSR used TEG4 as a gravity model, while for the TUM-orbit EIGEN-1S was used. Comparison with dynamic velocities from GFZ's Precise Science Orbit, for the same day, shows RMS of $0.08 \mathrm{~mm} / \mathrm{s}$ and $0.06 \mathrm{~mm} / \mathrm{s}$ for along and cross-track and $0.08 \mathrm{~mm} / \mathrm{s}$ for radial velocity component, respectively.

For more details about kinematic and dynamic POD we refer to Švehla and Rothacher $(2002,2003)$.

\subsection{Accelerometer data}

The accelerometer (ACC) data, as provided by GFZ, are given in a 10 seconds interval in a frame fixed to the spacecraft's body (SBF) together with attitude information. The attitude, which is given as quaternions, allows transformation between SBF and CIS. To integrate the accelerations along the orbit according to Eq. (3) either the orbit must be transformed to CIS, or the accelerations to CTS. Due to different sampling of ACC-data and TUM-orbit positions the latter are interpolated to a $10 \mathrm{~s}$ sampling.

It is known (see Reigber, 2001b), that due to a failure in one electrode of the accelerometer the measurements of the radial ACC-component are of bad quality. Recently an algorithm was found to overcome this problem, so the radial component of the ACC data can be reprocessed. Still this was not done for the data used in this paper and so the radial measurements are neglected at all. This seems to be acceptable, because the main part in $\triangle C$ comes from the ACC along-track component. From Eq. (3) it is obvious that forces acting orthogonal to the orbit do not contribute to $\Delta C$ at all and so accelerations in radial and cross-track direction can only enter by the order of misalignment of the SBF frame (measurement frame) with respect to the orbit frame. The deviation between both is below $2^{\circ}$.

Also the cross- and along-track components need to be calibrated due to unknown biases and scaling factors. The energy balance approach is highly sensitive to biased ACCdata, which makes it a powerful tool to estimate those biases. This holds especially for the along-track component (see next section). For the cross-track component the bias was not es- timated due to the low correlation between this component and $\Delta C$. Instead the time series of the cross-track component is reduced for it's mean value, which seems reasonable considering the satellites attitude. In all computations a scaling factor of 0.8 was used.

\section{Accelerometer calibration}

The ACC-data are expected to be very precise only within a certain measurement bandwidth. For lower frequencies outside this band larger errors can occur, which means that the accelerometer is biased by a long wavelength function or, at least, by a constant value. Due to the integration performed in Eq. (3) such a bias would appear as a drift in the potential. This way the energy balance approach allows the detection and estimation of accelerometer calibration parameters, which holds especially for the along-track bias, because, as already mentioned, it is the essential component in the nonconservative quantity $\Delta C$.

There are in principle two ways to determine the drift in the potential. One is to compare the observed potential to values derived from an independent potential model. The residuals consist of the bias, the propagation of further measurement and modelling errors as well as of imperfections of the reference model. The residuals of the disturbing potential derived from one day of CHAMP data with respect to the global model EIGEN-1S are shown in Fig. 3. There $T_{\text {uncomp. }}$ and $T$ represent the potential before and after compensation for $\Delta C$, respectively. From the decline of $T_{\text {uncomp. a loss }}$ of energy of around $160 \mathrm{~m}^{2} \mathrm{~s}^{-2}$ can be observed, which corresponds to a descent rate for CHAMP of around $25 \mathrm{~m}$ per day. Including $\Delta C$, we find a decline of around $2400 \mathrm{~m}^{2} \mathrm{~s}^{-2}$ per day, which can be explained by a bias in the along-track ACC-data. For an orbit radius of $6800 \mathrm{~km}$ CHAMP covers a distance of around $660000 \mathrm{~km}$ within his 15 revolutions per day. Using this distance in Eq. (3), from the given decline the bias can be estimated to be in the order of $3.6 \cdot 10^{-6} \mathrm{~m} \mathrm{~s}^{-2}$.

Another way of detecting the bias is to compare potential values at cross over points of the orbit tracks. Disregarding temporal variations in the gravity field, the potential values along the orbit must be identical at those points. Potential differences will show up, if the potential is drifting in time. Of course this works only if there is a crossing in threedimensional space or, as we have done here, if the potential is radially continued to a constant orbit height before. Within the used period CHAMP's altitude varies between 400 and $460 \mathrm{~km}$. For such a height variation the disturbing potential can be expanded in a Taylor series (restricted to quadratic order) with sufficient accuracy. Then the disturbing potential $T_{0}$ at constant (mean) height reads

$T_{0}=T-\left(\frac{\partial T_{0}}{\partial r} \Delta h+\frac{1}{2} \frac{\partial^{2} T_{0}}{\partial r^{2}} \Delta h^{2}\right)$,

where $\Delta h$ is the altitude difference between actual and mean orbit height. The series is expanded at mean height $h_{0}$ because then the radial derivatives in Eq. (6) can be taken from 


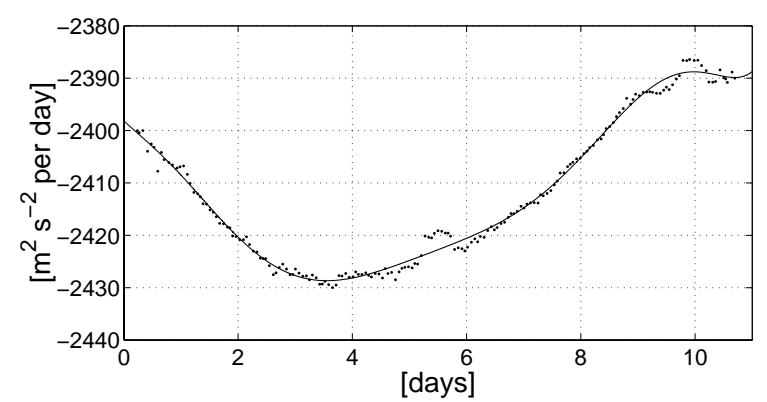

Fig. 4. Drift of disturbing potential at cross over points (dots) and a best fitting 9th order polynomial.

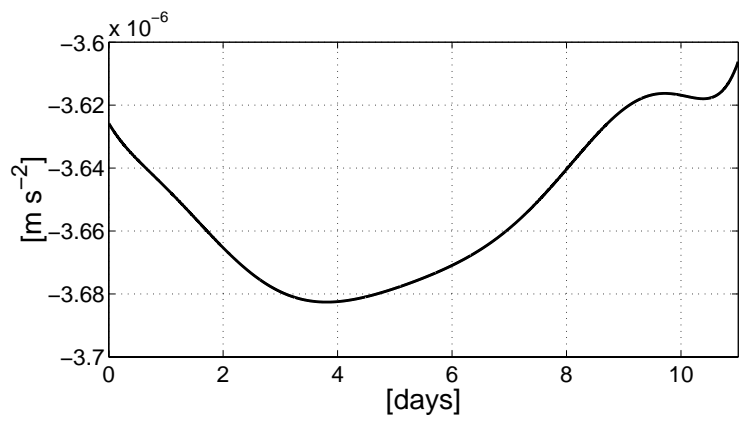

Fig. 5. Modelled behaviour of the ACC along-track bias over 11 days.

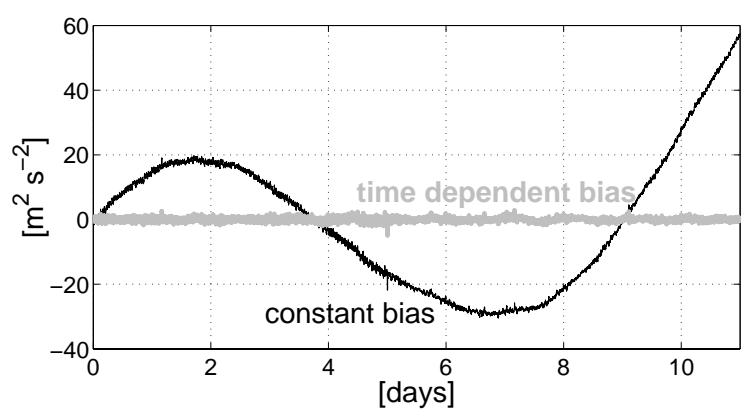

Fig. 6. Residual disturbing potential with respect to EIGEN-1S, considering a constant bias in ACC only, or taking a time dependent bias into account.

an a priori potential model and efficiently be computed in a global grid by spherical harmonic synthesis.

Once the drift is determined at the crossovers, the bias can be estimated. In Fig. 4 we have plotted the drift of the disturbing potential at the cross over points. There the drift is assigned to the mean epoch between the two corresponding tracks. The drift is at the level of around $-2410 \mathrm{~m}^{2} \mathrm{~s}^{-2}$ with variations of $\pm 20 \mathrm{~m}^{2} \mathrm{~s}^{-2}$. This means, that also the alongtrack bias is not constant in time, but shows long wavelength disturbances. We have fitted a 9 th order polynomial to the differences in Fig. 4, which means that also the bias can be modelled by a polynomial of the same order. The estimated bias-polynomial is shown in Fig. 5.

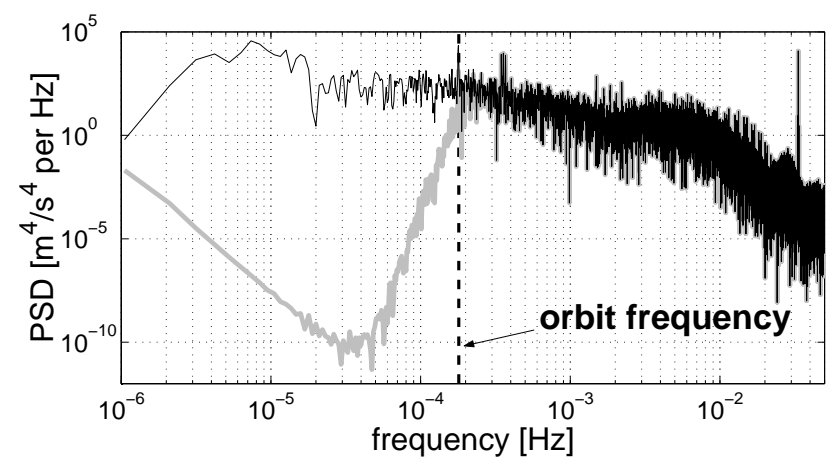

Fig. 7. Power spectral density (PSD) of the residual potential shown in Fig. 6 (black line). The gray line shows the same residuals after high-pass filtering below orbit frequency (indicated by the dashed line).

The bias in the along-track component shows variations of around $0.07 \mu \mathrm{m} \mathrm{s}^{-2}$ within 11 days, which is around $2 \%$ of the bias. The relevance of this result can be tested by comparing the disturbing potential to a potential model after bias correction. In Fig. 6 the residuals with respect to EIGEN-1S are shown, where in the first case (black line) only a constant bias is applied and in the second case (gray line) the biaspolynomial of Fig. 5 is used. This indicates that even though the estimated bias is quite stable (up to $2 \%$ ), still the effect of those variations on the potential cannot be neglected.

If one is not interested in calibration parameters, of course the long wavelength trend in the residual signal shown in Fig. 6 could also be reduced by high-pass filtering. The power spectral density of the residuals before and after filtering are shown in Fig. 7. There all frequencies below orbit frequency are filtered out. The result is equal to the one shown in Fig. 6. In any case, also all temporal variations in the signal are filtered out, not only variations of the accelerometer bias.

\section{Semi-analytical gravity field analysis}

The disturbing potential can be developed in a series of spherical harmonics. In gravity field analysis one wants to determine the coefficients $\Delta K_{l m}$ of such a series. This can be quite costly from the computational point of view, because the number of unknowns rises quadratically with the maximum degree of the series. Therefore we make use of the following semi-analytical approach (Sneeuw, 2000).

In case of special orbit geometry (constant inclination and radius) the normal matrix becomes block-diagonal and each block can be inverted separately. Thus the computational effort is lowered drastically and the analysis can be performed even on a usual PC within some minutes. The method can be used also for orbits, which are only near circular, like e.g. CHAMP, and approximation errors can be overcome by iterations. 


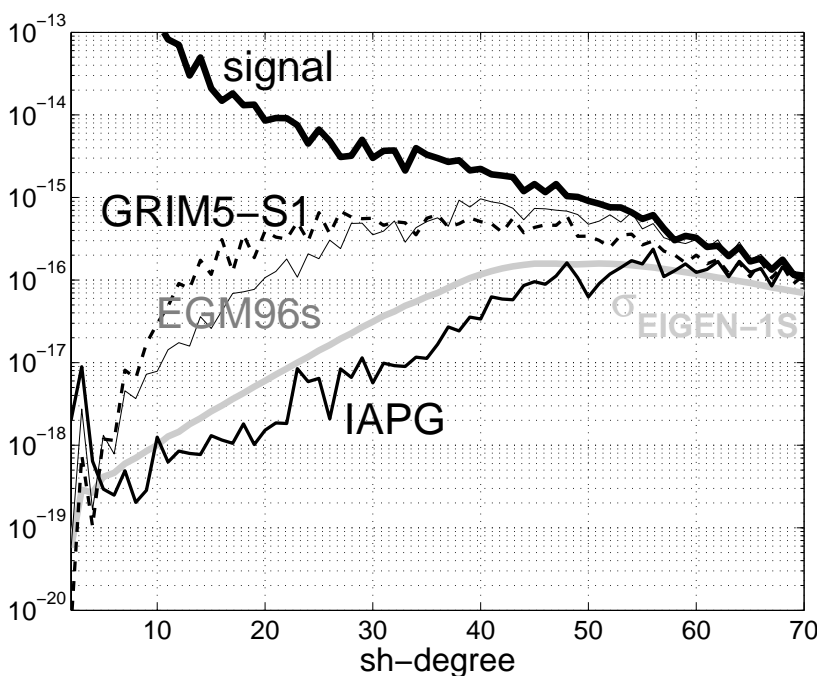

Fig. 8. Degree variances of EIGEN-1S (signal) and its differences to GRIM5-S1, EGM96s and the IAPG-solution. The difference between IAPG and EIGEN-1S is below the accuracy of EIGEN-1S (except for the very low coefficients), which means that the models are more or less identical.

In lumped coefficients representation the disturbing potential along the orbit is given by

$T(u, \Lambda)=\sum_{m=-L}^{L} \sum_{k=-L}^{L} A_{m k} e^{i(k u+m \Lambda)}$,

where $A_{m k}$ are the lumped coefficients, $\Lambda$ is the longitude of the ascending node and the argument of latitude along the orbit $u=v+\omega$ is the sum of true anomaly $v$ and the argument of perigee $\omega . L$ is the maximum spherical harmonic degree. The lumped coefficients $A_{m k}$ can then be connected to the potential coefficients $\Delta K_{l m}$ through

$A_{m k}=\sum_{l=\max (|m|,|k|)}^{L} \frac{G M}{R}\left(\frac{R}{r}\right)^{l+1} F_{l m k}(I) \Delta K_{l m}$,

where $F_{\operatorname{lmk}}(I)$ is an inclination function of constant inclination $I, R$ is the equatorial radius of the earth and $G M$ is the product of the earth's mass $M$ and the gravitational constant $G=6.67 \cdot 10^{-11} \mathrm{~m}^{3} \mathrm{~kg}^{-1} \mathrm{~s}^{-2}$. Take note that there appears no eccentricity function in Eq. (8) because the orbit is assumed to be circular (therefore also $r$ is constant). Equation (8) is the mathematical model used in a least squares adjustment to determine the unknown coefficients $\Delta K_{l m}$ from the quasi-observed lumped coefficients.

Equation 7 has the form of a two-dimensional Fourier series. If the disturbing potential along the orbit is known, the lumped coefficients can be computed from a twodimensional Fourier transformation. The proper space domain of a two-dimensional Fourier series is a torus (Hofmann-Wellenhof and Moritz, 1986). Therefore the potential along the orbit is first interpolated to a regular grid on the surface of a torus (see Sneeuw, 2001). This is straight forward as the potential is already reduced to constant orbit height in Eq. (5) and the torus coordinates $u$ and $\Lambda$ can be computed from the orbital elements. From the gridded potential the $A_{m k}$ are determined by 2D-FFT. Then the coefficients $\Delta K_{l m}$ can be computed by solving the system of linear equations in (8).

\section{Results}

From the disturbing potential along the 11-day orbit a set of spherical harmonic coefficients was derived. From this set a time series of disturbing potential was generated along the orbit. The residuals with respect to the original time series were again used to determine corrections to the first set of coefficients. This iteration was repeated until there was no reduction of the residuals anymore.

The result is compared to EIGEN-1S, which is a state-ofthe-art satellite-only model. As can be seen from the degree variances in Fig. 8 there is no signal above degree 60, or so. Therefore the following comparisons are restricted to this degree. In terms of geoid height our solution (denoted IAPG) and EIGEN-1S differ by up to $\pm 2 \mathrm{~m}$ (see Fig. 9, top). This is quite a good agreement compared to contemporary models like GRIM5-S1 (Gruber et al., 2000) or EGM96s (Lemoine et al., 1997). Both models show at least twice as large differences (see Fig. 9, middle and bottom). Also the structure of the differences is different. While the residuals of GRIM5S1 show a wave-like structure around the equator our solution shows a clear correlation to the orbit tracks. This could indicate orbit errors, probably velocity errors. The largest differences show up in regions of strong signal, like over the Andes or in east and south-east Asia.

From the degree variance plot (see Fig. 8) of the differences between the models it can be seen that, except for the very low coefficients, our solution is more or less identical to EIGEN-1S (the differences are well below the accuracy of EIGEN-1S), while there are significant differences to GRIM5-S1 and EGM96s. In RMS sense (see RMS values over latitude in Fig. 10) there are differences between the contemporary models of some decimeters up to meters in geoid height. Our solution fits to EIGEN-1S within a level of around $30 \mathrm{~cm}$.

\section{Concluding remarks}

The results in Sect. 6 show, that the gravity field determined from the energy balance approach fits better to the model EIGEN-1S than other contemporary satellite-only models like GRIM5-S1 or EGM96s. This proofs that the energy method is feasible for gravity field recovery. The solution achieved by Gerlach et al. (2003) using GFZ's RSO was at the level of around $20 \mathrm{~cm}$ at satellite altitude, while with the present TUM-orbit nearly the same accuracy was achieved at ground level. This significant improvement is due to the better quality of the orbits. 

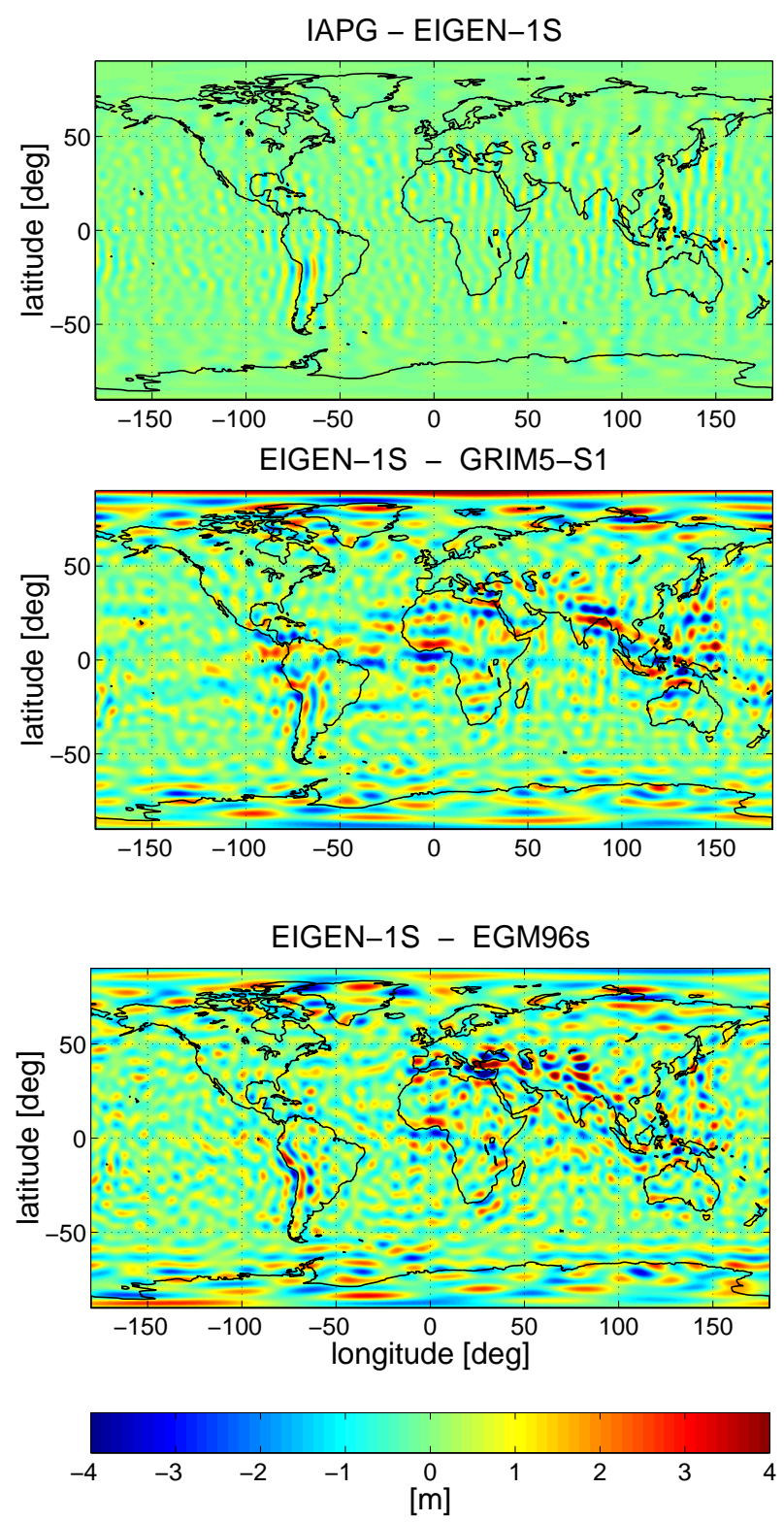

Fig. 9. Geoid height difference between the energy balance solution (denoted IAPG) and EIGEN-1S (top) and differences between EIGEN-1S and the models GRIM5-S1 (middle) and EGM96s (bottom).

As already mentioned, the reduced-dynamic approach to orbit determination is sensitive to the a priori gravity field. This holds especially for the velocity, which is the critical quantity in the energy integral (Visser et al., 2003). For the TUM-orbit the model EIGEN-1S was used. Considering this it should not be a surprise, that our solution fits better to EIGEN-1S than the other potential models. Obviously further investigations are necessary to decrease the correlation to the a priori field. The use of purely kinematic orbits could help because no prior information about the gravity field would be necessary. In this case however the outcome

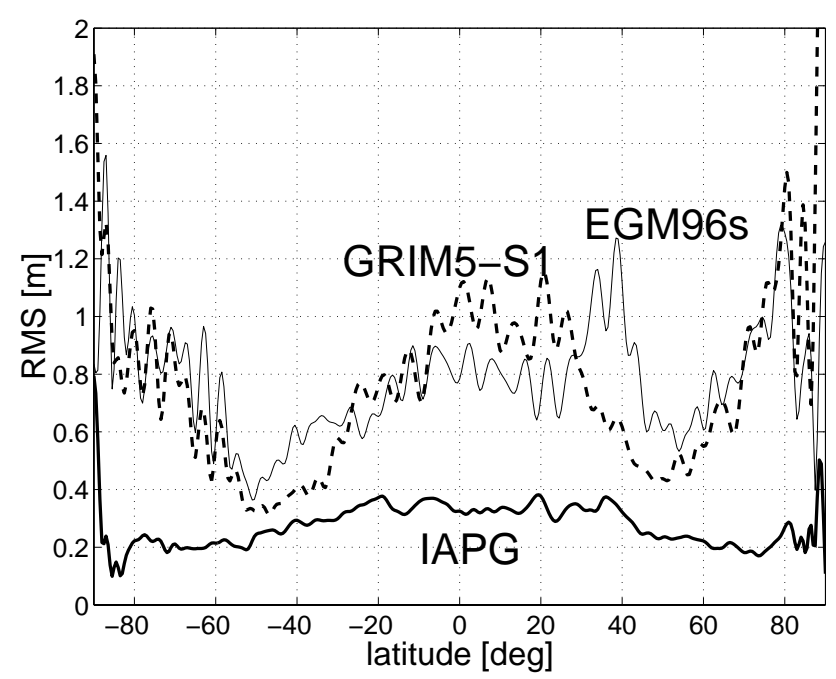

Fig. 10. RMS of geoid height difference to EIGEN-1S along parallels.

of the orbit determination are positions only and the velocities would have to be derived from those. This could be done, e.g., by differencing in time, fitting polynomials or spline interpolation.

Still the result shows, that the energy balance approach is a promising method for gravity field determination. It is possible to derive from only 11 days of continuous tracking of CHAMP a potential model which is (compared to the model EIGEN-1S) more accurate than the pre-CHAMP models, which are based on decades of data. It is also shown that the gravity field recovery can be done using an efficient semi-analytical approach. A full gravity field solution can be derived on a usual PC within some minutes.

Furthermore the cross-over analysis is a powerful tool to detect and determine calibration parameters for the accelerometer. In this paper we have only estimated the bias of the along-track component because the dissipative term $\Delta C$ is most sensitive to this component, while the radial and cross-track components play a minor role. It must be mentioned that using the cross-over analysis not only the accelerometer bias (including its variations) but also the neglected radial component, errors in the scaling factors (we have used 0.8 for all components) as well as all kinds of temporal variations enter our computation. The same holds for all effects which are not modelled. All those effects are somehow mapped into the bias estimation. Considering the direct luni-solar tidal potential which is by far the largest temporal variation, it amounts to up to $6 \mathrm{~m}^{2} \mathrm{~s}^{-2}$. If we compare the residual potential with respect to a constant bias (see Fig. 6), it reaches values of $60 \mathrm{~m}^{2} \mathrm{~s}^{-2}$, which is one order of magnitude larger than direct tidal effects. If we neglect all temporal variations (including tides) our estimate for the bias variations would be wrong for around $10 \%$. Making up only $2 \%$ of the bias, this error in the bias variation would lead to an error of the bias of only $0.2 \%$. Therefore it can be 
assumed that un-modelled temporal variations are not significant to our current accuracy level. On the other hand an error in the scaling factor would result in a similar effect as a temporal variation in the along-track bias. Further investigations are necessary to overcome this problem and to decouple bias and scaling factors. Also the radial ACC-component should be included in future computations, now that the erroneous data can be corrected.

Acknowledgements. We are grateful to GeoForschungsZentrum Potsdam for providing CHAMP data.

\section{References}

Bjerhammar, A.: A new approach to Satellite Geodesy, USAEGI MRADA, Alexandria, Virginia, 1967.

Gerlach, Ch. and Sneeuw, N.: Schwerefeldbestimmung mit einem semi-analytischen Ansatz unter Ausnutzung der Energieerhaltung. Paper presented to Geodätische Woche, Potsdam, October, 2000.

Gerlach, Ch., Sneeuw, N., Visser, P. N. M. A., and Švehla, D.: CHAMP gravity field recovery with the energy balance approach: first results, in: First CHAMP Mission Results for Gravity, Magnetic and Atmospheric Studies, (Eds) Reigber, Ch., H. Lühr, P. Schwintzer, Springer-Verlag, 2003.

Gruber, Th., Bode, A., Reigber, C., Schwintzer, P., Balmino, G., Biancale, R. and Lemoine, J.-M.: GRIM5-C1: Combination solution of the global gravity field to degree and order 120, Geophys. Res. Lett., 27 (24), 4005-4008, 2000.

Guthmann, A.: Einführung in die Himmelsmechanik und Ephemeridenrechnung, Spektrum Akademischer Verlag, 2000.

Hedin, A. E.: Extension of the MSIS Thermosphere Model into the Middle and Lower Atmosphere, J. Geophys. Res., 96, 1159, 1991.

Hofmann-Wellenhof, B. and Moritz, H.: Introduction to spectral analysis. In: Mathematical and numerical techniques in physical geodesy, Sünkel, H. (ed.), Lecturs Notes in Earth Sciences, Vol. 7, Springer-Verlag, 1986.

Hotine, M. and Morrison, F.: First integrals of the equation of satellite motion, Bulletin Géodésique, No. 91, 41-45, 1969.

Howe, E. and Tscherning, C. C.: Preliminary analysis of CHAMP state vector and accelerometer data for the recovery of the gravity potential, in: First CHAMP Mission Results for Gravity, Magnetic and Atmospheric Studies, (Eds) Reigber, Ch., H. Lühr, P. Schwintzer, Springer-Verlag, 2003.

Ilk, K. H.: Formulierung von Energieaustauschbeziehungen zur Ausmessung des Gravitationsfeldes, In: Die Arbeiten des Sonderforschungsbereichs 78 Satellitengeodäsie der Technischen Universität München im Jahre 1982, Schneider, M. (ed.), Astronomisch-Geodätische Arbeiten der Bayerischen Kommission für die Internationale Erdmessung, No. 43, 1983.

Ilk, K. H.: Energy Relations for the Motion of two Satellites within the Gravity Field of the Earth, Paper presented to Geodätische Woche, GFZ Potsdam, October 2000.
Jacobi, C. G. J.: Über ein neues Integral für den Fall der drei Körper, wenn die Bahn des störenden Planeten kreisförmig angenommen und die Masse des gestörten vernachlässigt wird, Monthly Reports of the Berlin Academy of Science, July 1836.

Jekeli, Ch.: The determination of gravitational potential differences from satellite-to-satellite tracking, Celestial Mechanics and Dynamical Astronomy, 75, 85-101, 1999.

Lemoine, F. G., Smith, D. E., Kunz, L., Smith, R., Pavlis, E. C., Pavlis, N. K., Klosko, S. M., Chinn, D. S., Torrence, M. H., Williamson, R. G., Cox, C. M., Rachlin, K. E., Wang, Y. M., Kenyon, S. C., Salman, R., Trimmer, R., Rapp, R.H. and Nerem, R. S.: The development of the NASA GSFC and NIMA Joint Geopotential Model, In: Gravity, Geoid and Marine Geodesy, IAG Symposia, Segawa, J., Fujimoto, H., Okubo, S. (eds), Vol. 117, 461-469, Springer-Verlag, 1997.

McCarthy, D. D.: IERS Conventions 1996, IERS Technical Note $21,1996$.

O'Keefe, J. A.: An application of Jacobi's integral to the motion of an earth satellite, Astron. J. 62(8), 266-267, 1957.

Reigber, Ch.: Zur Bestimmung des Gravitationsfeldes der Erde aus Satellitenbeobachtungen, Deutsche Geodätische Kommission, Reihe C, No. 137, 1969.

Reigber, Ch., Schwintzer, P., Koenig, R., Neumayer, K.-H., Bode, A., Barthelmes, F., Foerste, C., Balmino, G., Biancale, R., Lemoine, J.-M., Loyer, S. and Perosanz, F.: Earth gravity field solutions from several months of CHAMP satellite data, Eos Trans AGU, 82(47), Fall Meeting Suppl. G41C092, 2001a.

Reigber, Ch.: CHAMP newsletter, no 4, accessible via http://op. gfz-potsdam.de/champ/more/newsletter $\backslash$ _CHAMP $\backslash$ _004.html, $2001 b$.

Schneider, M.: Beiträge zur Bahnmechanik künstlicher Erdsatelliten, Teil II, Deutsche Geodätische Kommission, Reihe C, No. 113, 1967.

Sneeuw, N.: A semi-analytical approach to gravity field analysis from satellite observations, Deutsche Geodätische Kommission, Reihe C, No. 527, 2000.

Sneeuw, N.: Dynamical satellite geodesy on the torus: Blockdiagonality from a semi-analytical approach, In: Gravity, Geoid and Geodynamics 2000, Sideris, M.G. (ed.), IAG Symposia, Vol. 123, Springer-Verlag, 2001.

Švehla, D. and Rothacher M.: Kinematic Orbit Determination of LEOs Based on Zero- or Double-Difference Algorithms Using Simulated and Real SST Data, In: Vistas for Geodesy in the New Millenium, Adam, J., Schwarz, K.P. (eds), IAG Symposia, Vol. 125, 322-328, Springer-Verlag, 2002.

Švehla, D. and Rothacher, M.: CHAMP double-difference kinematic orbit with ambiguity resolution, in: First CHAMP Mission Results for Gravity, Magnetic and Atmospheric Studies, (Eds) Reigber, Ch., H. Lühr, P. Schwintzer, Springer-Verlag, 2003.

Torge, W.: Geodesy, Walter de Gruyter Verlag, 1980.

Visser, P. N. A. M., Sneeuw, N., and Gerlach, C.: Energy integral method for gravity field determination from satellite orbit coordinates, J. Geodesy, in print, 2003.

Wolff, M.: Direct Measurements of the Earth's Gravitational Potential using a Satellite Pair, J. Geophys. Res., 74(22), 5295-5300, 1969. 